\title{
Multidisciplinary treatment of breast cancer under COVID-19 pandemic
}

\author{
Pin Gao", Shiheng Li", Ying Jin, Zhimin Fan \\ Department of Breast Surgery, the First Hospital of Jilin University, Changchun, China \\ Contributions: (I) Conception and design: Z Fan; (II) Administrative support: Z Fan; (III) Provision of study materials or patients: Y Jin; (IV) \\ Collection and assembly of data: P Gao, S Li; (V) Data analysis and interpretation: S Li, P Gao; (VI) Manuscript writing: All authors; (VII) Final \\ approval of manuscript: All authors. \\ "These authors contributed equally to this work. \\ Correspondence to: Zhimin Fan. Department of Breast Surgery, the First Hospital of Jilin University, Changchun 130021, China. \\ Email: fanzhimn@163.com.
}

\begin{abstract}
Background: The COVID-19 pandemic broke out and spread across the whole world rapidly in the early 2020. To minimize exposure risk and preserve medical resources, multidisciplinary treatment (MDT) of breast cancer has been adjusted.
\end{abstract}

Methods: We collected breast cancer patients visited our department during January to April in 2019 $(\mathrm{n}=495)$ and $2020(\mathrm{n}=346)$ separately. Information including patient characteristics, admission process and treatment strategies were obtained.

Results: MDT during COVID-19 Pandemic significantly changed. Among those patients in 2020, the proportion of young patients ( $<35$ years old) and neoadjuvant chemotherapy increased, intervals of adjuvant and neoadjuvant chemotherapy prolonged and the hospitalization length was shortened.

Conclusions: Although long-term follow-ups are necessary to identify the outcomes, current alternative and adjustment made for COVID-19 not only decreased infectious risk, but also made full use of hospital resources and optimized breast cancer patient management.

Keywords: Breast cancer; COVID-19 pandemic; multidisciplinary treatment (MDT); treatment strategy

Received: 09 July 2020; Accepted: 14 September 2020; Published: 31 October 2020.

doi: $10.21037 /$ tbcr-20-38

View this article at: http://dx.doi.org/10.21037/tbcr-20-38

\section{Introduction}

In early 2020, 2019 novel coronavirus disease (known as COVID-19) broke out and became a public health emergency of international concern. COVID-19 spread around the whole world in a short time because of the various transmission routes (such as droplets, contact, aerosol and other means) and long incubation period (1). Jilin Province (a province in northeastern China) launched the first-level response to major public health emergencies rapidly on January 25 th, 2020. Since health care workers and hospitals are at high risk of infection, we could see many changes made in medical industry after the breakout of COVID-19. In the First Hospital of Jilin University, we pretested triage of fever patients to identify suspected cases at the first time and admitted reservation registration only through the internet. In respond to COVID-19, experts of different areas also updated guidelines for different diseases. According to Liang's research (2), cancer patients seems to be more suspicious to COVID-19 in China. Breast cancer is known as the most common cancer among women. To combat COVID-19 and find optimal ways of diagnosis and treatment under the epidemic situation, professors in our Department of Breast Surgery in the First Hospital of Jilin University made some adjustment towards the management of breast cancer according to the new rules of our hospital and guidelines of breast cancer during the outbreak of COVID-19. We present the following article in accordance with the 
MDAR reporting checklist (available at http://dx.doi. org/10.21037/tbcr-20-38).

\section{Methods}

This study was an observational real world study. The research was approved by the Ethics Committee of the First Hospital of Jilin University (2020-398) and was conducted in accordance with the Helsinki Declaration of 1964 (as revised in 2013). Informed consent was obtained from all individual participants included in the study.

\section{Patient data}

This research collected all the breast cancer inpatients treated in the Dept of Breast Surgery of the First Hospital of Jilin University. The total number of inpatients from Jan $1^{\text {st }} 2019$ to Apr $30^{\text {th }} 2019$ was 495 . The total number of inpatients from Jan $1^{\text {st }} 2020$ to Apr $30^{\text {th }} 2020$ was 346.

\section{Inclusion and exclusion criteria}

To be included in this research, all cases must meet the following inclusion criteria: (I) the patient had to be received treatment in the Dept of Breast Surgery of the First Hospital of Jilin University from Jan $1^{\text {st }} 2019$ to Apr $30^{\text {th }} 2019$ or Jan $1^{\text {st }} 2020$ to Apr $30^{\text {th }} 2020$. (II) The aims of hospitalization included neoadjuvant chemotherapy, postsurgery chemotherapy, surgery and surgery concomitant with the first cycle of chemotherapy.

Furthermore, cases that met the following criteria were excluded: (I) the breast cancer patient received treatment in other departments of the First Hospital of Jilin University. (II) The patient was not diagnosed as breast cancer. (III) The aims of hospitalization were not belonging to those above. (IV) The patient rejected the appropriate treatment come up with doctors.

\section{Observational end point}

The first end point was pre-hospital evaluation including admission access and patients' age and distribution. The second end point was treatment strategy including treatment decisions, surgical methods, hospitalization length and cost, time intervals between treatment.

\section{Statistics analysis}

We used $t$-test to compare whether the difference of end points above in 2019 and 2020 is statistically significant $\left(\mathrm{P}<0.0001,{ }^{* * * *} ;\right.$ ns, not significant). The softwares we used are GraphPad Prism 6.0c, Excel 2011, IBM SPSS statistics Version 26.

\section{Results}

\section{Pre-hospital evaluation}

\section{Changes of admission process}

When compared to the same time of last year, the admission process of breast cancer patients in our department changed a lot (Figure 1). In the year of 2019, after getting the examination of breast ultrasound and mammography, susceptive breast cancer patients could be admitted into hospital and then receive further examinations such as biopsy, immunohistochemistry and other routine examinations. Once the patient was diagnosed as breast cancer, he/she would get neoadjuvant chemotherapy or surgery according to the clinical stage and molecular classification of breast cancer. However, during the outbreak of COVID-19, all the examinations related to the diagnosis of breast cancer should be finished at outpatient department and only breast cancer patients could be admitted into hospital. Furthermore, blood routine test and lung CT image are necessary for excluding COVID-19. Before entering inpatient department, nurses will monitor the temperature of both the patients and accompanying visitors, inquiry contact history and help them sign related forms.

\section{Changes of age and distribution}

During the epidemic of novel pneumonia, the age (Figure 2) and distribution of breast cancer patients visiting our department also changed. The age trend of 2020 is similar to that of 2019. The patients in the group between 45 to 54 years old, which are usually of high risk of breast cancer, are more than other age groups. However, when compared to 2019 , there are more young patients $(<35)$ and less old patients $(>65)$ in 2020 . The distribution of patients' regions in 2020 is similar to that in 2019 . However, the diversity of out-of-province patients' districts decreased during COVID-19 pandemic because of the limitation of provincial trip. Therefore, in the period of COVID-19 pandemic, most patients came from Jilin province and the number of out-of-province patients is almost zero expect for Heilongjiang and inner Mongolia (data not shown). 
Admission process of 2019
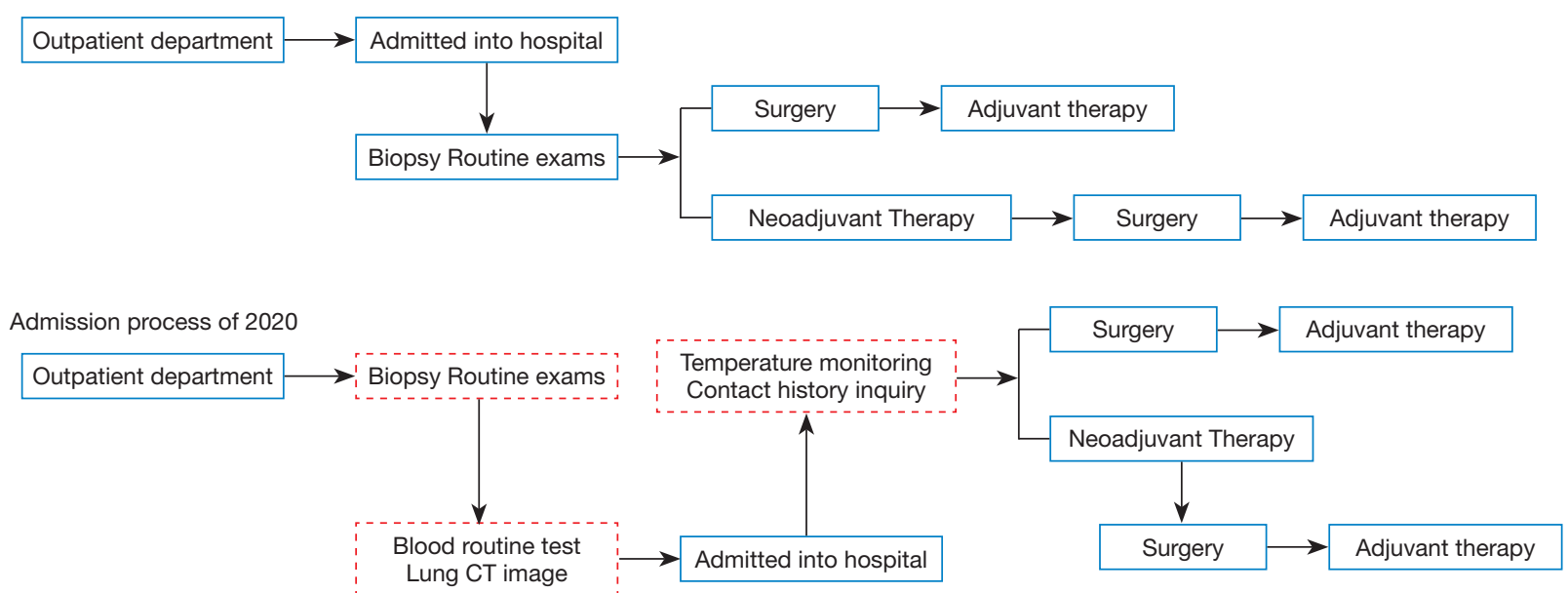

Figure 1 The differences of admission process in 2019 and 2020.

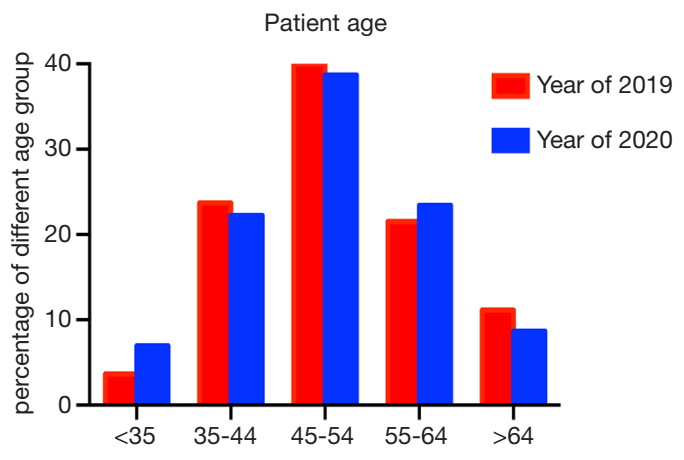

Figure 2 The differences of inpatient ages in 2019 and 2020.

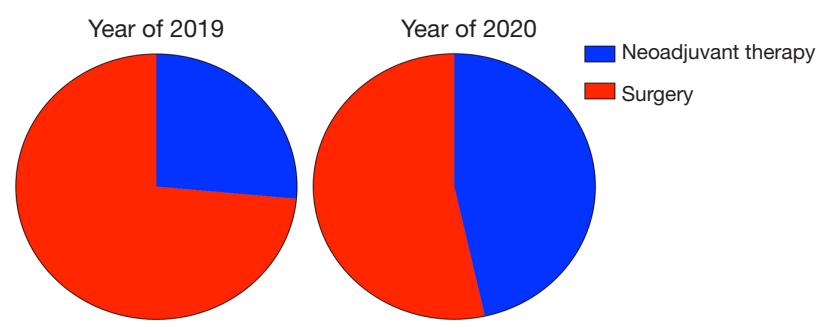

Figure 3 The differences of treatment decisions in 2019 and 2020.

\section{In-bospital treatment}

\section{Changes of treatment decision}

As shown in Figure 3, there were 344 breast cancer patients newly diagnosed in our department during January and April, 2019. Among those, 91 patients (26.54\%) accepted neoadjuvant therapy, including neoadjuvant chemotherapy and neoadjuvant endocrine therapy. The other 253 patients $(73.55 \%)$ accepted surgery directly. During the same time of 2020 , only 77 patients (53.47\%) accepted surgery. The percentage of neoadjuvant therapy increased to $46.53 \%$.

In the first four months of 2019, there were 307 patients accepted surgery, including the patients who finished neoadjuvant therapy. Among those patients, 20.52\% accepted breast-conserving surgery, $1.63 \%$ accepted phase I breast reconstruction after mastectomy, the other $73.55 \%$ patients accepted mastectomy directly. During the same time of 2020 , only 140 patients finished surgery. The percentage of breast conserving surgery and phase I reconstruction decreased to $17.41 \%$ and $0.72 \%$, and more patients $(82.14 \%)$ chose breast mastectomy (Figure 4).

\section{Changes of hospitalization length and cost}

The hospital length of 2020 is much shorter than that of 2019. As shown in Figure 5, for neoadjuvant therapy patients, the average hospitalization days of 2019 and 2020 were 6.19 and 2.30 (Figure 6). The average length of stay of surgery patients was 14.62 days in 2019 and in 2020 , the number decreased to 7.66 days. For patients accepted adjuvant chemotherapy, the average length of stay in 2019 and 2020 were 5.84 and 2.54 days, separately. Some patients accepted the $1^{\text {st }}$ adjuvant chemotherapy after surgery without being discharged. The hospital stay of those patients was also shorten from 17.62 days in 2019 to 12 days in 2020 .

As with the changes of diagnosis and treatment process, the cost also changed. In 2020, the average hospitalization 


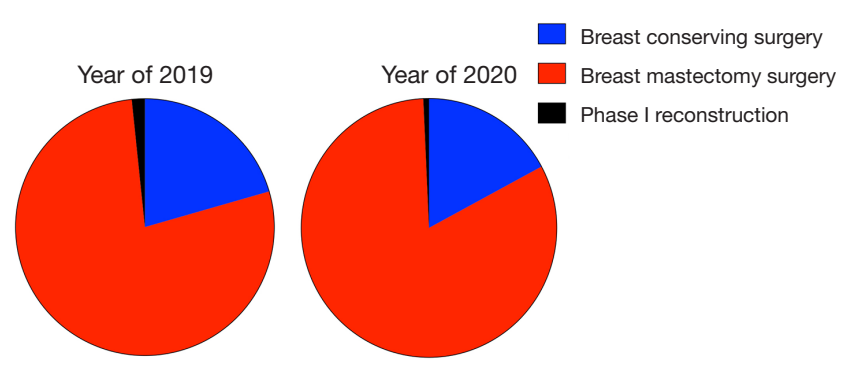

Figure 4 The differences of surgical methods in 2019 and 2020.

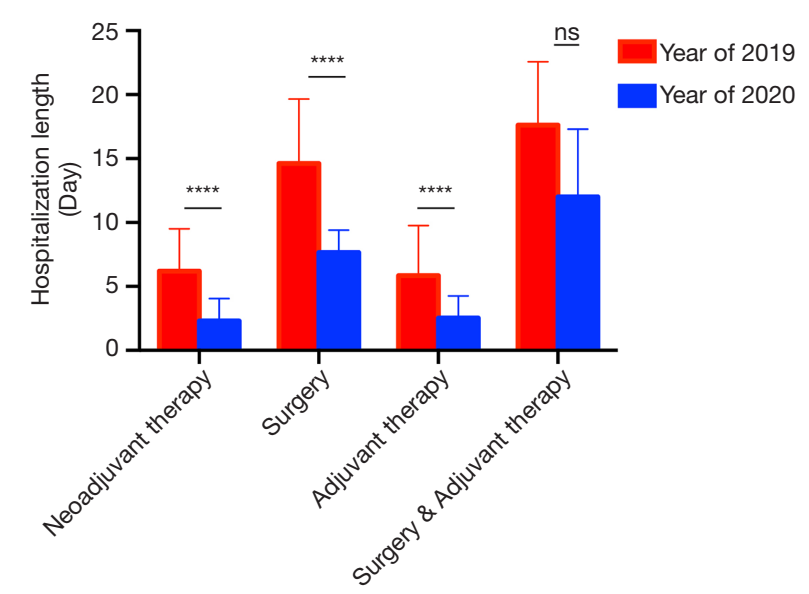

Figure 5 The differences of hospitalization lengths in 2019 and 2020. ****, $\mathrm{P}<0.0001$; ns, not significant.

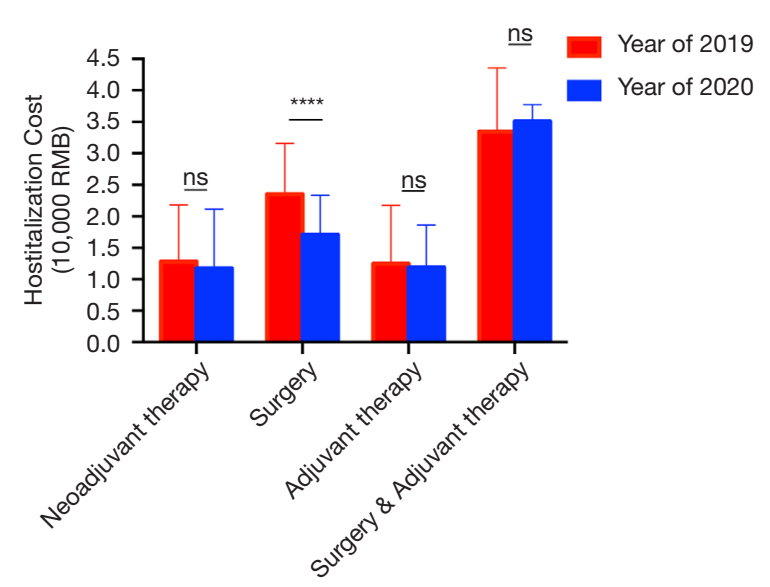

Figure 6 The differences of hospitalization costs in 2019 and 2020. ****, $\mathrm{P}<0.0001$; ns, not significant.

cost was RMB 17,100 for those accepted surgery, which was much lower than that of 2019, RMB 23,500 (Figure 6). However, the average cost of other patients who came to our department for neoadjuvant therapy or adjuvant therapy did not change a lot.

\section{Changes of time intervals between treatments}

There were 88 patients who received surgery following the first chemotherapy from Jan $1^{\text {st }} 2019$ to Apr $30^{\text {th }} 2019$. Meanwhile, only 3 cases were calculated in 2020. Time interval to initial chemotherapy was 8 to 15 days, and the peak value was 12 days in 2019. While time interval from surgery to initial chemotherapy ranged from 3 to 7 days (Figure 7A). When it came to days between final neoadjuvant chemotherapy and surgery, there were 51 patients in 2019 who received surgical care 21 to 30 days later. In 2020, 41 patients got surgery after 11 to 20 days. However, delay of $>40$ days from final neoadjuvant chemotherapy to surgery happened on 6 patients which accounted for $15 \%$ of total (Figure 7B). Compared with that potion of patients in 2019, there was no delay of $>40$ days. Furthermore, intervals between cycles of chemotherapy in 2019 were $19.49 \pm 0.2466$ days, while those in 2020 were $32.60 \pm 1.679$ days (Figure $7 C$ ). In term of neoadjuvant chemotherapy time intervals, it was $18.94 \pm 0.3737$ days in 2019 compared with $33.02 \pm 1.420$ days in 2020 (Figure 7D).

\section{Discussion}

During epidemic of COVID-19, there were less patients visited our department for various reasons: isolation for migration history, registration limitation, and even outpatient appointment registration. The change of patient flow leads to better hospital environment, shorter time of examinations and longer time for communication with clinicians. For the inpatients, it is necessary to exclude the infection of COVID-19 before being admitted to hospital through contact history inquiry and related examinations. Some prevention measures such as compulsory mask wearing and safety distance not only decrease the risks of infecting COVID-19, but also other diseases spread through respiratory tract. As we can see, the changes during epidemic made a better hospital environment for both patients and medical workers.

In the special time, less outpatients came to visit our department. Different from old breast cancer patients, both the number and percentage of young patients are higher than last year. Part of the reason is that younger women care more about their health these days and young age is a factor of poor prognosis. The change of admission process is another important reason. Registration online only and 
A

Time from surgery to initiation of adjuvant chemotherapy

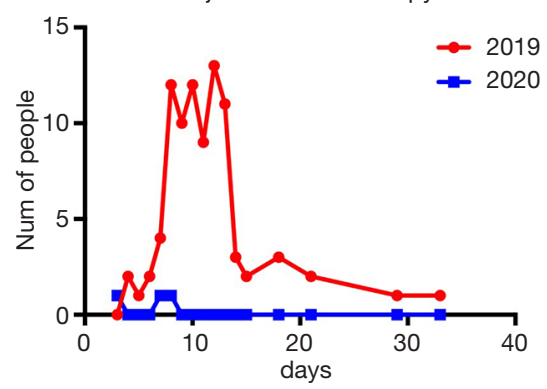

C
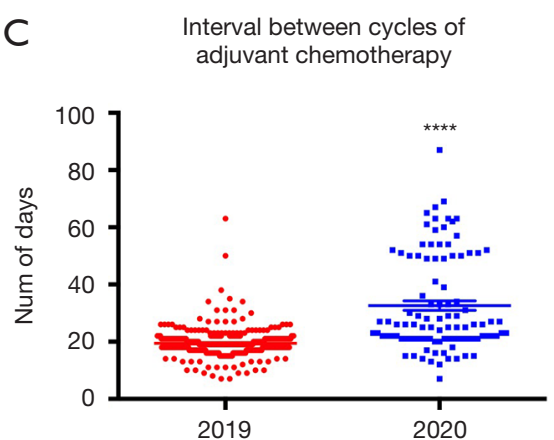

B

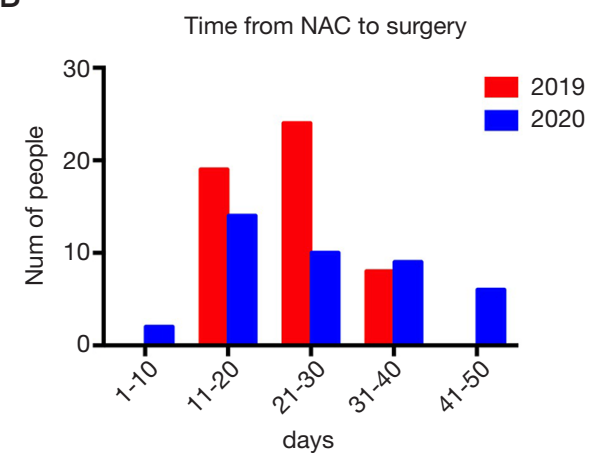

D Interval between cycles of NAC

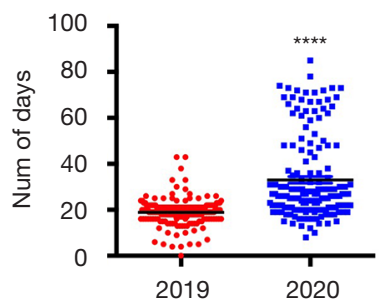

Figure 7 The differences of chemotherapy in 2019 and 2020 (A) time from surgery to initiation of adjuvant chemotherapy. (B) Time from neoadjuvant chemotherapy to surgery. (C) Interval between cycles of adjuvant chemotherapy. (D) Intervals between cycles of NAC. ****, $\mathrm{P}<0.0001$.

limitation of accompany made it difficult for elder patients asking for medical help. When it came to distribution of patient regions, the proportion of patient regions of Jilin Province did not change much, but the number of out-ofprovince patients reduced, which indicated that COVID-19 pandemic had a big impact on the regions of patients. When implementing new measures, such as electronic management instead of traditional management model in the hospital, we need to meet the needs and consider the abilities of different levels and ages of people.

The guidelines for managing breast cancer patients changed during the COVID-19 pandemic. Breast cancer experts recommend to expand neoadjuvant therapy indication (3). In the past, the purpose of neoadjuvant therapy was to achieve surgical resection for inoperable disease or to downstage cancer to facilitated conserving surgery. In order to minimize exposure risk of COVID-19, neoadjuvant therapy is used for earlier breast cancer these days. For non-invasive breast cancer, such as ductal carcinoma in situ (DCIS) with no risk of invasion, a delay of operation until after COVID-19 is acceptable. For
ER (estrogen receptor) positive DCIS and early invasive carcinoma, neoadjuvant endocrine therapy is a good choice. For triple negative breast cancer (TNBC) patients, neoadjuvant chemotherapy is more suitable and for HER2 (human epidermal growth factor receptor) overexpression patients, targeted therapy is also necessary. Neoadjuvant therapy not only decreased the risk of COVID-19, but also help clinicians evaluate therapeutic effects. On the other hand, when the outcome of one patient accepting neoadjuvant therapy is PCR (pathologic complete response), maybe she would have a chance to avoid breast cancer operation. Clinicians need more time to follow up, and get the conclusion whether the change of therapeutic decisions during COVID-19 is good for breast cancer prognosis. If neoadjuvant therapy could provide an alternative to surgery, there will be no doubt that conserving the whole breast is a protection for both physical and mental health of breast cancer women.

Since breast cancer patients finished all preoperative examinations before being admitted into hospital, the average hospitalization day in 2020 was much shorter 
than that in 2019. As the length of stay decreased, the risk of contact with other inpatients and infection decreased. On the other hand, the adverse effects of chemotherapy or surgery did not increase. Even during non-epidemic period, it brings benefits to patients and hospitals when optimizing treatment process, increasing bed occupancy rates, shortening average hospitalization length. The changes made for COVID-19 are also good for optimizing health resources, making full use of outpatient resources and improving hospital operating efficiency (4).

During COVID-19 pandemic, the strategy of recruiting patients has been modified so that many breast cancer patients have to prolong the time of treatment which include time from surgery to start of adjuvant chemotherapy, intervals between final cycle of neoadjuvant chemotherapy and surgery, time intervals between cycles of adjuvant or neoadjuvant chemotherapy. Our research showed that time from surgery to initiation of chemotherapy mostly was $8-15$ days in 2019 . However, time interval was 3-7 days in 2020. According to evidence-based medical research, patients who received adjuvant chemotherapy after surgery took less 30-40\% risk compared with those who did not (5). But the optimal time from surgery to first cycle of chemotherapy is still controversial. Several clinical trials demonstrated that shorter delay (6) ( $<4$ weeks versus $>8$ weeks) of adjuvant chemotherapy decreased recurrence rate and overall survival of breast cancer (7). Therefore, it suggested that it was better to operate adjuvant chemotherapy as early as possible. Based on our clinical data, patients would initiate adjuvant chemotherapy within 1 week during COVID-19 pandemic in order to reduce the infectious odds, which may decrease recurrence rate in all subtypes of breast cancer. On the other hand, patients under adjuvant chemotherapy could not come to hospital on time because of trip restriction and adjustment of inpatient strategy. Compared to that in 2019, average time intervals between cycles of adjuvant chemotherapy were delayed to 32 days. The common standard of intervals between cycles is 3 or 4 weeks. But more and more researches focused on study of dose-dense chemotherapy in order to explore more efficient regimens. According to Norton-Simon Hypothesis, it is more efficient to shorten adjuvant chemotherapy cycle intervals (8) than to increase dose of chemotherapeutic drug (9). What is more, it is helpful to avoid chemotherapy resistance. From evidence above, longer delay of adjuvant chemotherapy will harm the benefit from chemotherapy, so we recommend that it is better to decrease the hospitalization length in order to reduce the risk of COVID-19 exposure.

Data between Jan $1^{\text {st }} 2019$ and April $30^{\text {th }} 2020$ showed interval from last cycle of neoadjuvant chemotherapy to surgery most concentrated on 21-31 days, while that in 2020 most focus on 11-20 days. From our data, this portion of patients decreased about $20 \%$ during the COVID-19 pandemic compared to number in 2019. The potential reason why the interval between last cycle of neoadjuvant chemotherapy to surgery is shorter during COVID-19 pandemic is that the total number of patients in our department reduced and that patients with neoadjuvant chemotherapy usually had lymph node metastasis. Those should have priority of surgery in some degrees. When comes to intervals between cycles of neoadjuvant chemotherapy, it prolonged from 20 days in 2019 to 33 days in 2020 . However, there is still no exact results showing what is the optimal interval setting. It is also necessary to study the more efficient chemotherapeutic regimens based on current chemotherapeutic drugs.

\section{Conclusions}

COVID-19 pandemic has both bad and good impacts on the strategy of breast cancer treatment. On the one hand, women diagnosed as breast cancer have to postpone the previous treatment and face with risk of complexity of hospitalization and virus exposure. On the other hand, adjustment of treatment strategy brings new ideas into the management and care of breast cancer. For example, we can reduce hospitalization length and intervals from neoadjuvant chemotherapy to surgery as well as increase the utility of outpatient care. Our research will support the changes of treatment progress in the future and provide some clinical data.

\section{Acknowledgments}

Funding: None.

\section{Footnote}

Reporting Checklist: The authors have completed the MDAR reporting checklist. Available at http://dx.doi.org/10.21037/ tbcr-20-38

Data Sharing Statement: Available at http://dx.doi. org/10.21037/tbcr-20-38 
Conflicts of Interest: All authors have completed the ICMJE uniform disclosure form (available at http://dx.doi. org/10.21037/tbcr-20-38). The authors have no conflicts of interest to declare.

Etbical Statement: The authors are accountable for all aspects of the work in ensuring that questions related to the accuracy or integrity of any part of the work are appropriately investigated and resolved. The study was conducted in accordance with the Declaration of Helsinki (as revised in 2013). This research was approved by the Ethics Committee of the First Hospital of Jilin University (2020398). Informed consent was obtained from all individual participants included in the study.

Open Access Statement: This is an Open Access article distributed in accordance with the Creative Commons Attribution-NonCommercial-NoDerivs 4.0 International License (CC BY-NC-ND 4.0), which permits the noncommercial replication and distribution of the article with the strict proviso that no changes or edits are made and the original work is properly cited (including links to both the formal publication through the relevant DOI and the license). See: https://creativecommons.org/licenses/by-nc-nd/4.0/.

\section{References}

1. Deng J, Chen X, Zhang J, et al. Risk Control of Subjects and Investigators in Clinical Trial of Anti-cancer Drug

During the Epidemic of COVID-19. Anti-tumor Pharmacy 2020;10:48-52.

2. Liang W, Guan W, Chen R, et al. Cancer patients in

doi: $10.21037 /$ tbcr-20-38

Cite this article as: Gao P, Li S, Jin Y, Fan Z. Multidisciplinary treatment of breast cancer under COVID-19 pandemic. Transl Breast Cancer Res 2020;1:28.
SARS-CoV-2 infection: a nationwide analysis in China. Lancet Oncol 2020;21:335-7.

3. Dietz JR, Moran MS, Isakoff SJ, et al. Recommendations for prioritization, treatment, and triage of breast cancer patients during the COVID-19 pandemic. the COVID-19 pandemic breast cancer consortium. Breast Cancer Res Treat 2020;181:487-97.

4. Su Z, Lin J, Wang Y, et al. Practice and Discussion on the Average Length of Stay Under New Medical Reform. Chinese Hospital Management 2018;38:72-3.

5. Early Breast Cancer Trialists' Collaborative Group (EBCTCG), Peto R, Davies C, et al. Comparisons between different polychemotherapy regimens for early breast cancer: meta-analyses of long-term outcome among 100,000 women in 123 randomised trials. Lancet 2012;379:432-44.

6. Yu KD, Fan L, Qiu LX, et al. Influence of delayed initiation of adjuvant chemotherapy on breast cancer survival is subtype-dependent. Oncotarget 2017;8:46549-56.

7. Liu ZY, Jin Y, Fan ZM. Timing and delays in early-stage breast cancer treatment during outbreaks of Corona Virus Disease 2019. Chinese Journal of Practical Surgery 40:250-4.

8. Henderson IC, Berry DA, Demetri GD, et al. Improved outcomes from adding sequential Paclitaxel but not from escalating Doxorubicin dose in an adjuvant chemotherapy regimen for patients with node-positive primary breast cancer. J Clin Oncol 2003;21:976-83.

9. de Almeida FK, Rosa DD. Adjuvant Dose-Dense Chemotherapy for Breast Cancer: Available Evidence and Recent Updates. Breast Care (Basel) 2018;13:447-52. 